\title{
Investigation of transport property of fibrous media: 3D virtual modeling and permeability calculation
}

\author{
Xiang Huang ${ }^{1,2} \cdot$ Yanwei Zhao $^{1} \cdot$ Hongwei Wang $^{3} \cdot$ Hao Qin $^{3} \cdot$ Donghui Wen $^{1} \cdot$ \\ Wei Zhou ${ }^{4}$
}

Received: 3 June 2016 / Accepted: 23 February 2017 / Published online: 13 March 2017

(C) The Author(s) 2017. This article is published with open access at Springerlink.com

\begin{abstract}
Porous Metal Fiber Sintered Sheet (PMFSS) shows a significant potential in the development of highperformance and compact fuel cell. To achieve optimized PMFSS structural design, it is essential to evaluate permeability, i.e., the correlation between fiber structures and the transport property. To perform pre-scale simulation, a method is proposed in this research to construct 3D virtual PMFSS models using morphological features extracted from X-ray images. A length-weighted orientation method is used to evaluate the anisotropy of fiber arrangement in the through-thickness direction, and a numerical model is proposed to evaluate the flow property through the gaps between fibers. Simulation results confirm that the Forchheimer law dominates flow behavior as flow rate rises. Permeability of both the transverse and the parallel flow directions are investigated and the simulation data obtained are compared with results obtained from various sources such as the analytical equations in the literature, numerical calculations based on the Lattice Boltzmann Method (LBM) as well as material testing experiments. It is found in the comparison that the permeability results obtained in this
\end{abstract}

Hongwei Wang

Hongwei.Wang@port.ac.uk

Xiang Huang

522250912@qq.com

1 School of Mechanical Engineering, Zhejiang University of Technology, Hangzhou 310014, China

2 George W. Woodruff School of Mechanical Engineering, Georgia Institute of Technology, Atlanta, GA 30332, USA

3 School of Engineering, University of Portsmouth, Portsmouth PO1 3DJ, UK

4 Department of Mechanical and Electrical Engineering, Xiamen University, Xiamen 361005, China work are consistent with the values predicted by the analytical models of layered fiber arrangement proposed by Spielman and Goren. The proposed method thus provides an efficient way of PMFSS design optimization using virtual models.

Keywords Porous metal · Permeability · Anisotropy · Virtual fiber network

\section{Introduction}

Porous Metal Fiber Sintered Sheet (PMFSS), a new kind of functional porous material, has been successfully produced recently and proved to be promising [1]. It has interconnected high porosity, large specific surface, and good mechanical strength [2]. These advantages have led to its successful applications to fuel cells as the catalyst support and the gas diffusion layer [3, 4]. These applications involve some key components in which mass and charge transport take place and as such the transport property of fibrous media is of paramount importance. Understanding of the correlation between the transport property and pore scale morphology can provide important guidelines for optimizing material design and achieving improved performance in fuel cells. In particular, to better derive this correlation, effective, and practical modeling method, reflecting the actual fiber structures is of significant importance.

To address this challenge and reduce the experimental process-synthesis-characterization cycle for PMFSS structural optimization [5], this paper presents an effective and efficient methodology to develop 3D stochastic parameterized models of fibrous media constructed using structural information extracted from X-ray images and on this basis investigates the transport properties at high flow rates. 
Therefore, the microstructure generation process can be directly integrated with the morphology and transport characterization analyses. More precisely, morphology analysis of PMFSS structure is first undertaken and a method for constructing 3D virtual models is developed. Second, a method is proposed to enable integrated model construction and flow simulation through coupling CAD and Computational Fluid Dynamics (CFD) software packages. On this basis, simulation experiments are conducted to evaluate these methods and undertake further analysis of permeability to provide guidelines for PMFSS structural optimization before fabrication.

The rest of the paper is organized as followed. Section 2 reviews relevant studies on model construction, transport property simulation, and permeability analysis. After this, Sect. 3 explains the virtual simulation methodology and details the methods developed in this research. Section 4 describes the evaluation of the methods by comparing the simulation results to those obtained using existing methods. Finally, the main conclusions are drawn in Sect. 5.

\section{Related research}

A lot of research has been done to explore flow laws in a fibrous network. Stylianopoluos et al. [6] used finite-element simulation to study permeability in fibrous material with isotropic and oriented fiber arrangements. Specifically, aligned networks were generated by selecting directional vectors from an anisotropic distribution and the impact of porosity on permeability was discussed. Nabovati et al. [7] reconstructed fibrous media with straight cylinders of random arrangement and studied the permeability using the lattice Boltzmann method. Structural features involving curvature and aspect ratio of fibers on the permeability were also determined. The virtual geometric models $[6,7]$, however, were constructed without sufficient verification by comparing to the real structures. Recently, X-ray tomography has rapidly developed and has been widely applied to material science, especially in the non-destructive reconstruction of porous materials, such as granular materials [8], metal foams [9], and fiber materials [10]. Pradeep et al. [11] converted 3D tomography images into pore network modeling $[12,13]$ which was composed of pores and connecting pore throats and in which a mass balance was imposed at each pore, and the flow through the pore throats was approximated. Using 3D image data, Koviu et al. [15] studied the permeability of plastic felt and hand-sheet paper. In this work, simulation results (using the lattice Boltzmann method and the finite-difference method) were compared with experimental results. The lattice Boltzmann method was performed on 3D tomographic images directly. To generate grid data for the finite-difference method from
3D images, the Geodict package (http://www.geodict.com) was used $[5,14]$. Similar work was reported by Brun et al. [16], in which flow laws were explored in metal foams. The reconstructed faceted geometries in STereo Lithography (STL) file format were volumetrically meshed into the continuous fluid domain and simulation was conducted using a common Computational Fluid Dynamics (CFD) software package. However, meshing disordered 3D geometries from STL data are usually very time-consuming as it requires extensive computational resources [17]. Therefore, it is promising to develop an alternative virtual modeling method and fit the model to the real geometrical characteristics that can be obtained through morphological exploration from 3D tomographic images. Such virtual models are usually constructed with Computer-Aided Design (CAD) packages in which the models can be exported using file formats supported by the most commercial CFD software, such as Fluent Inc. In a recent study, Heitzmann et al. [18] investigated the permeability of anisotropic sintered metallic fiber structures through CFD simulation. In this study, the anisotropy tensor was applied for geometric model fitting and the analytical method by Shou et al. [19] was used to obtain the best results. In this way, optimal structure achieving the best performance can be determined by slightly modifying the geometric model through altering the controllable structural parameters and investigating their corresponding performances through simulation experiments.

It should be noted that that, in most publications, numerical results of the transport property are investigated at creep flow (low Reynolds number). Unfortunately, due to technical limitations [17, 33], the experimental settings are usually performed at higher flow rates at which the impact of inertial effects cannot be neglected. However, the numerical results of transport properties of porous material at higher flow rates are seldom reported [16]. To fill these gaps, this research aims to develop a method for constructing virtual models which can support effective simulationbased study of transport properties at high flow rates.

\section{Methods}

\subsection{Morphology analysis of PMFSS}

The methodology of manufacture and microstructural characterization of PMFSS have been fully described in our previous studies [35]. In general, after cutting continuous copper fibers and subsequent sintering and generating PMFSS, the 3D images of PMFSS can be obtained through X-ray tomography. A group of image processing which involves selection of ROI, image enhancement of anisotropic diffusion [20], and binarization is undertaken, and then, the 
skeleton extraction is performed to obtain a topologically identical representation of the fiber structure. Finally, various morphological statistical distributions involving fiber segment lengths, radius, types, 3D orientations, and tortuosity are performed based on the skeleton segments. Figure 1a shows the optical photograph of PMFSS with $90 \%$ porosity, and Fig. 1b shows the skeleton segments distinguished in different colors inside the reconstructed 3D geometries using the Marching Cubes algorithm [21].

In addition, the porosity of PMFSS is known in advance during manufacture using the mass-volume method as follows [1]:

$E(\%)=\left(1-\frac{M_{\mathrm{p}}}{\rho_{\mathrm{c}} V_{\mathrm{p}}}\right) \times 100$.

In Eq. (1), $V_{\mathrm{p}}$ is the volume of PMFSS (observation window); $M_{\mathrm{p}}$ represents the mass of PMFSS; and $\rho_{\mathrm{c}}$ denotes the density of red copper. The porosity value is obtained during image binarization.

(a)

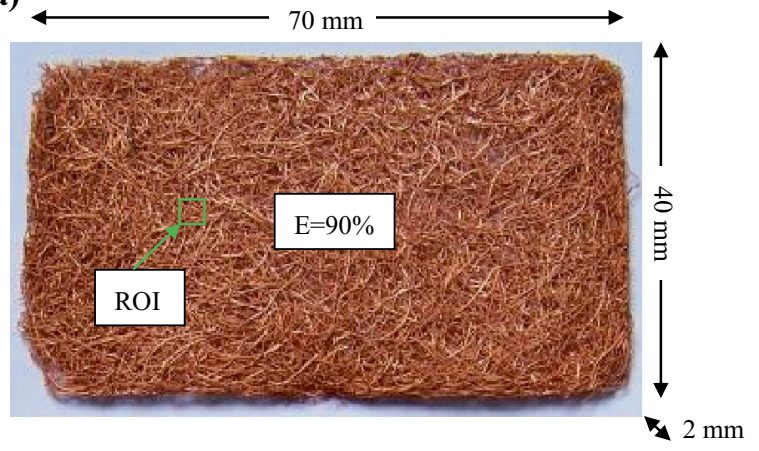

(b)

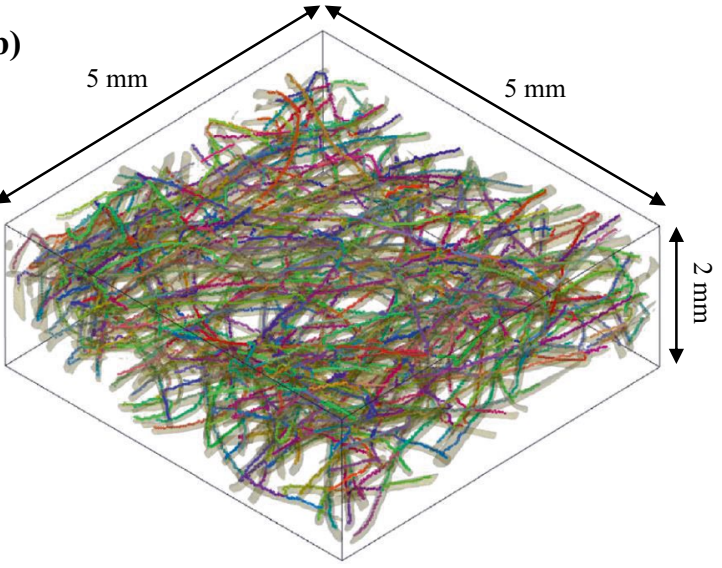

Fig. 1 a Optical photograph of PMFSS with $90 \%$ porosity sizing $40 \times 70 \times 2 \mathrm{~mm}$; b skeleton segments distinguished by different colors inside the $3 \mathrm{D}$ reconstructed geometry of ROI with a size of $5 \times 5 \times 2 \mathrm{~mm}$

\subsection{Generation of 3D virtual PMFSS}

The virtual model should have the capability to mimic the actual fiber structures particularly their orientations. In this work, the orientation parameter is depicted in spherical coordinates with a thickness angle $\theta(0<\theta<\pi / 2)$, and a material plane angle $\varphi(0<\varphi<2 \pi)$, respectively. Analogous to most fibrous media [22, 23], fibers in PMFSS are randomly distributed in the material plane with anisotropy observed in the through-thickness direction. Various methods have been developed to model the anisotropy of fiber orientation, such as the anisotropy tensor $[6,18]$ and the specific distribution (e.g., uniform distribution) which have a constant mean value [28]. In this work, the anisotropy of fiber arrangements is described by the probability density of the direction in polar coordinates, as described in [24]:

$p(\theta, \varphi)=\frac{1}{4 \pi} \frac{\beta \sin \theta}{\left(1+\left(\beta^{2}-1\right) \cos ^{2} \theta\right)^{3 / 2}}$

where $\beta$ is the anisotropy parameter which describes the anisotropy of the orientation. The trend of being layered aggravates when the value of $\beta$ increases.

The orientation statistic data are obtained according to the fiber segments [35]. However, the virtual models are constructed as the accumulation of stacked single fibers rather than fiber segments divided by sintered joints. Unfortunately, because fibers are tortuous and bonded together, it is quite a challenge to trace and identify single fiber from complex fibrous networks. Thus, an alternative method is applied to evaluate the orientation of a single fiber from fiber segments weighted by the segment lengths. This method is called the length-weighted orientation method. Assume that there are $n$ fiber segments $f(i),(i=1, \ldots, n)$ in some orientation regions, after the length-weighted operation, number $n$ is changed into $N$ as follows:

$N=\sum_{i=1}^{n} \frac{\operatorname{len}(f(i))}{\text { meanlen }}$

In Eq. (3), len $(f(i))$ represents the length of fiber segment $f(i)$, and meanlen is the average length of fiber segments. Figure 2 illustrates the principle of the length-weighted orientation method.

It is assumed that fiber (a) and fiber (b) are of equal length and fiber (a) is divided into four segments by three sintered joints. Before the length-weighted operation, the radio of fiber numbers in the two orientations is $4: 1$. After the conversion process, however, the updated value is $(3+1+2+1) /(14 / 5): 7 /(14 / 5)=1: 1$.

Finally, the fitting $\beta$ values are determined by slightly increasing $\beta$ values by 0.1 at each step until they reach the lowest accumulated frequency error of the experimentally measured length-weighted orientation data. Consequently, 
(a)

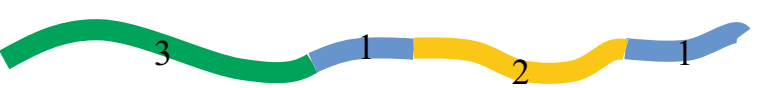

(b)

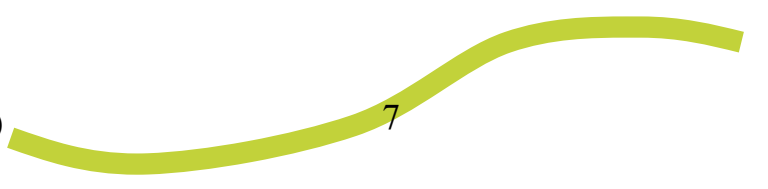

Fig. 2 Illustration of length-weighted orientation: a fiber with length of 7; $\mathbf{b}$ fiber divided into four segments with lengths of 3,1,2, and 1

as shown in Fig. 3, the fitted values of $\beta$ for the PMFSS structures with 80 and $90 \%$ porosities are 2.7 and 5.4, respectively.

To generate the virtual models suitable for simulation, the method proposed by Fassel et al. [10] is used, which constructs a periodic elementary volume with parameters describing fiber length, thickness, position, orientation, and curvature. First, a random position vector is generated within a unit cell. Then, an orientation vector is determined following the distribution function described using Eq. (2). The director line is later extended along the assigned orientation vector in both directions evenly until it reaches the fiber length. To determine crimp, some control points are generated at fixed interval $\Delta h$ around the director line. They are defined by a distribution vector $\Delta r$ at the plane orthotropic to the director line (Fig. 4a) and are subsequently connected using the spline line Application Programming Interface (API) provided by SolidWorks. The tube-like single fiber is finally generated by sweeping the spline line with circular cross section of a certain radius. This single fiber generation process is repeated several times until the desired porosity is obtained, which can be evaluated in the mass attribute menu in SolidWorks. At last, to form the periodic unit, fiber parts outside the unit cell are cut and moved to their opposite sides (Fig. 4b, c). Figure $4 \mathrm{~d}$ shows the ensemble of four units, which obviously indicates the periodicity of the unit cell. It is noteworthy that the overlap of fibers is allowed as this is similar to the effect of sintering during manufacture.

\subsection{Flow laws and simulation}

It is well known that flow at low flow rates (small Reynolds number) is governed by Darcy's law:

$-\frac{\mathrm{d} P}{\mathrm{~d} l}=\frac{\mu}{k} u$.

In Eq. (4), $\mathrm{d} P / \mathrm{d} l$ is the pressure gradient along the flow region of length $l ; \mu$ is the viscosity; $u$ is the superficial velocity; and $k$ is the permeability of the media. As the flow rate rises, the effect of inertia turns to be non-negligible,
Fig. 3 Determination of fitting values of parameter $\beta$ through comparison of the fitting data and the experimental results of length-weighted orientations
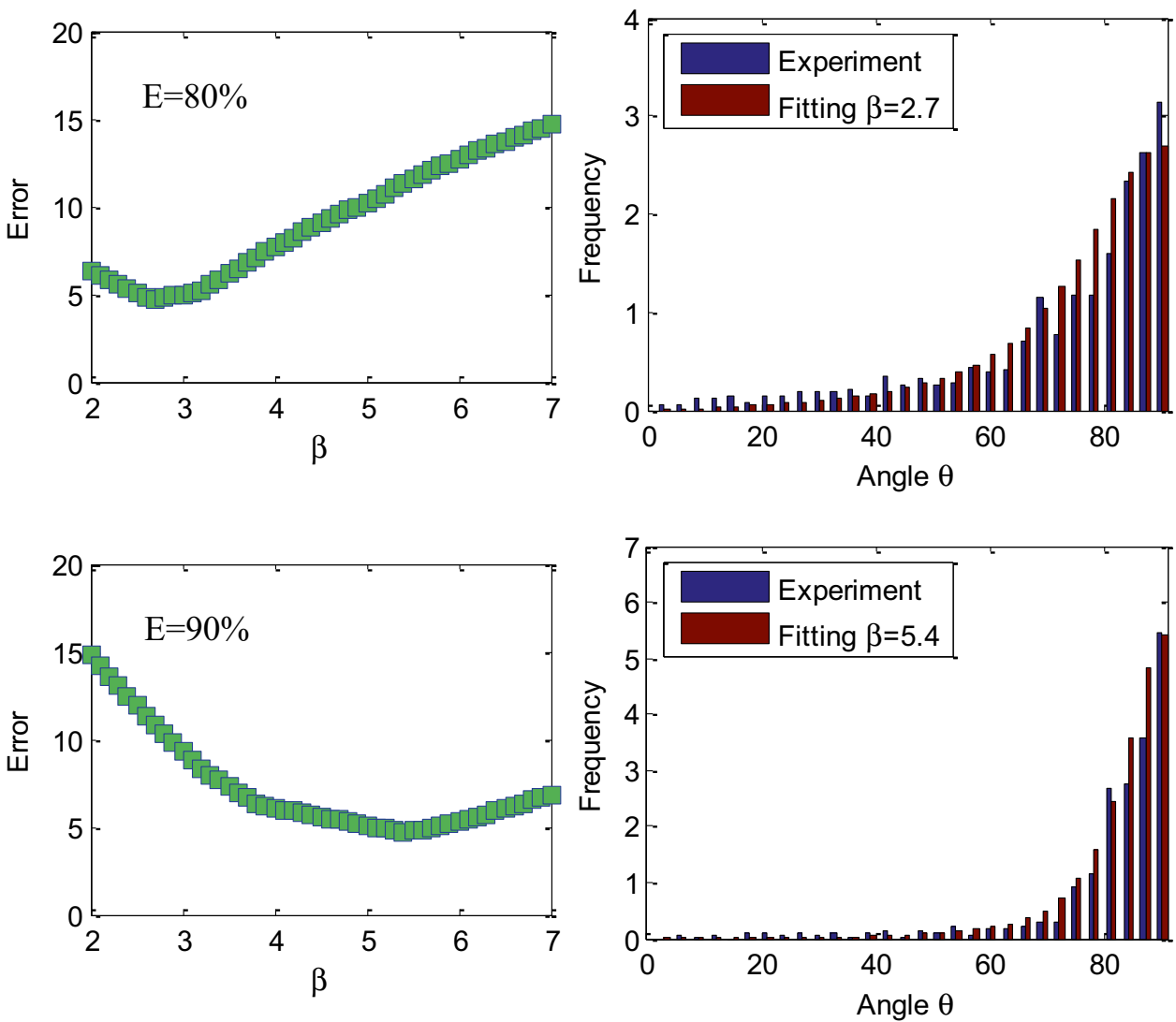


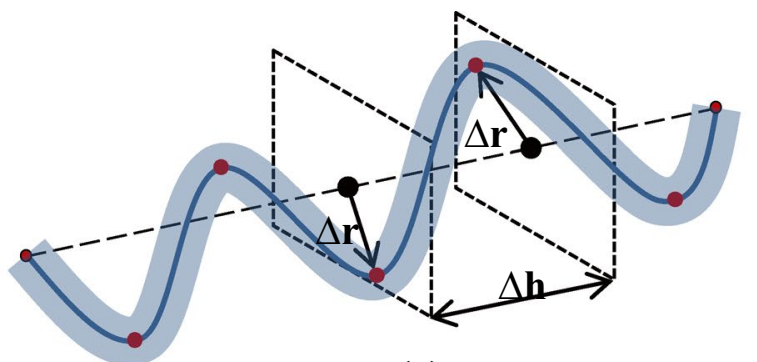

(a)

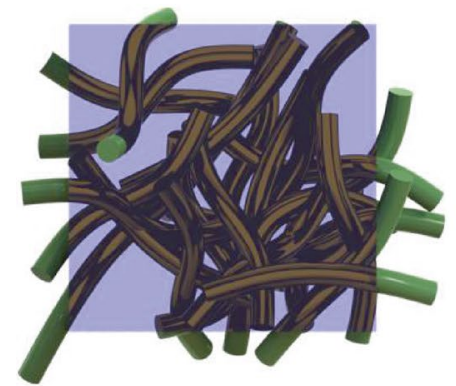

(b)

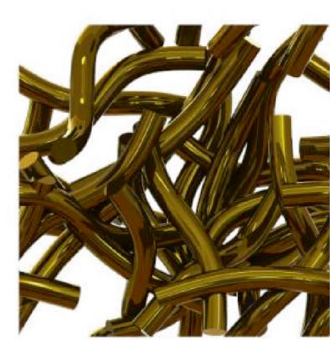

(c)

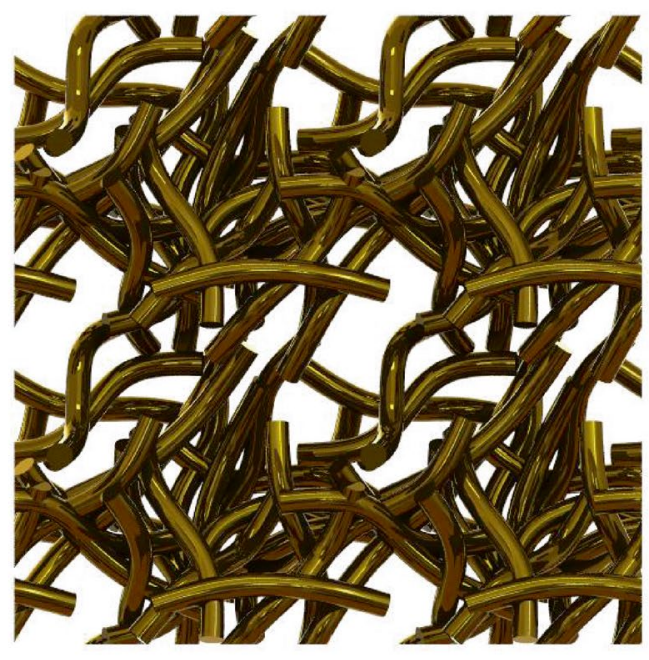

(d)

Fig. 4 Generation of a 3D fiber network: a generation of a single fiber; $\mathbf{b}$ fibers cut by a unit; $\mathbf{c}$ cut fibers moved to its corresponding faces with one periodic unit generated; $\mathbf{d}$ demonstration of periodicity of four units

and the linear relationship between the pressure drop and the flow rate turns to be nonlinear. Therefore, the empirical Forchheimer equation is then used to account for the deviation from Darcy's law:

$-\frac{\mathrm{d} P}{\mathrm{~d} l}=\frac{\mu}{k} u+k_{1} \rho u^{2}$.

In this equation, $\rho$ is the density of the fluid and $k_{1}$ is the inertial parameter or the non-Darcy flow coefficient [25].

In addition, the Reynolds number is defined as

$R e=\frac{\eta L}{\mu}$.

In Eq. (6), $L$ is the characteristic length of the flow zone and $\eta$ is the mass flux density. The most widely accepted characteristic length is the pore diameter. It is determined by the mean radius (chosen to be 282 and $385 \mu \mathrm{m}$ for PMFSS structures with 80 and $90 \%$ porosities, respectively) of the balls that fill the gaps between the fibers, i.e., the void phase [34]. The transport property of the same material was experimentally explored in [33] using nitrogen $\left(\mathrm{N}_{2}\right)$ gas (viscosity $\left.1.663 \mathrm{e}-5\right)$ with the maximal flow velocity $3.57 \mathrm{~m} / \mathrm{s}$, and thus, the deduced Reynolds number according to Eq. (6) is 69 and 94 for PMFSS structures with 80 and $90 \%$ porosities, respectively. These values are bigger than 10 which are referred as the upper limit for Darcy's law. Moreover, the validity of Forchheimer equation [i.e., Eq. (5)] at such higher flow rates was verified during simulation.
The simulation domain of the gaps between fibers was obtained by subtracting the virtual fiber network from the surrounding cubic space processed in SolidWorks (Fig. 5 a, b). To obtain the appropriate domain size, i.e., the Representative Volume Element (RVE) [16], the Brinkman screening length criterion [26] is introduced. According to the definition of Brinkman screen length, a 3D cubic box with the size of $14 \sqrt{k}$ by side length is big enough to smooth the effect of local inhomogeneity. The parameter $k$ represents permeability which can be estimated in advance using Jackson and James's equation [27]:

$\frac{k}{r^{2}}=\frac{3}{20 \phi}[-\ln (\phi)-0.931]$.

In Eq. (7), $\phi$ represents volume fraction parameter and equals $1-E$, and $\frac{k}{r^{2}}$.is the dimensionless permeability with $r$ representing fiber's radius. According to Eq. (7), the predicted permeability of the PMFSS structure with $90 \%$ porosity is $5.1 \mathrm{e}-9 \mathrm{~m}^{2}$. In addition, the size of RVE according to $14 \sqrt{k}$ is $980 \mu \mathrm{m}$, which is approximate ten times the size of the fiber diameter $(100 \mu \mathrm{m})$. In this paper, to satisfy the Brinkman screen length criterion, the side length of RVE chosen is about 12 times the size of the fiber diameter. The RVE side length is also taken as the length of the director line during generation of single fiber. Consequently, the aspect ratio (fiber length/diameter) is bigger than 12:1 after bending. It is reported by Nabovati et al. [7] that the aspect ratio is not important for permeability with aspect ratio bigger than 6 . The RVE size for the PMFSS 

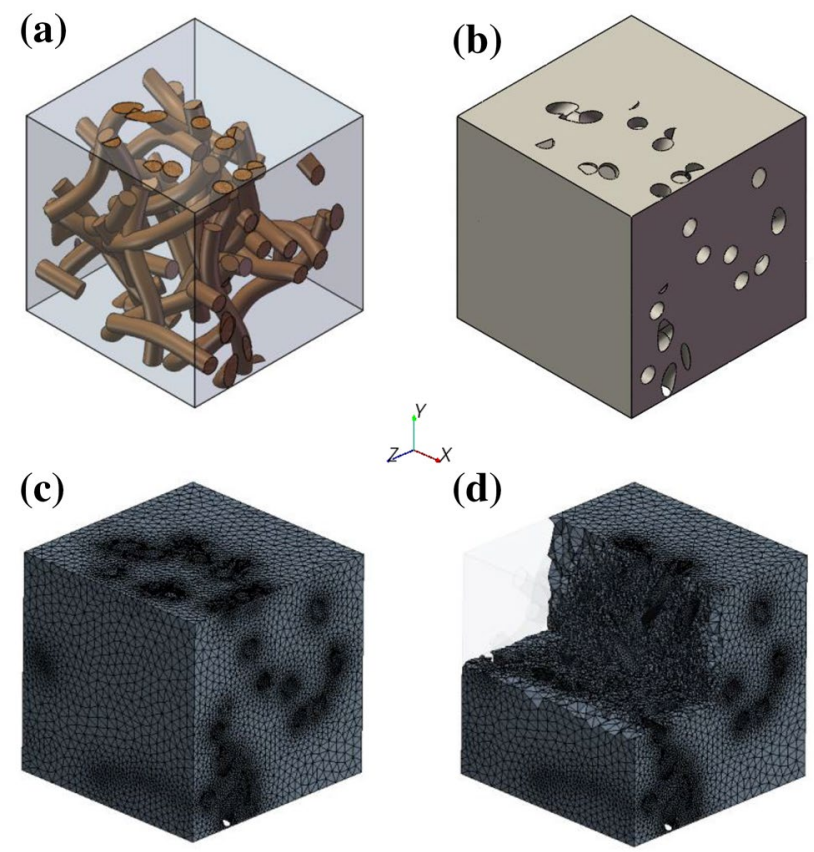

Fig. 5 Generation of a simulation model; a initial network; b void space model; c generated mesh; and $\mathbf{d}$ look into the mesh volume

structure with $90 \%$ porosity is also applicable for PMFSS of lower porosity, as the permeability decreases with the decrease of porosity.

Finally, the model is imported to a commercial Computational Fluid Dynamics (CFD) package (i.e., Fluent) which can mesh it for simulation. The governing Navier-Stokes equations are solved using the finite volume CFD code from Fluent, Inc. During simulation, pressure boundary conditions are applied to the inlet and outlet. No-slip boundary condition is applied to the surface of fibers, and symmetry boundary condition on the lateral faces. A group of pressure drops is imposed and the results are used to depict the pressure gradient versus velocity curves.

\section{Results}

\subsection{Mesh convergence study}

To ensure the numerical results are not mesh dependent. In this respect, the number of cells is increased by increasing the grid points on the cross section of the fibers gradually. In particular, the number of grid points on the cross section is increased from 5 to 20 , and consequently, the number of cells in the 3D model is increased from approximately 50,000-3,000,000. Virtual PMFSS with $90.2 \%$ porosity of physical size $(1.25 \mathrm{~mm})^{3}$ is thus constructed. Pressure drop of $20 \mathrm{~Pa}$ is applied in the through-thickness direction (z-axis), and the flux is reported in Fluent. Figure 6 shows

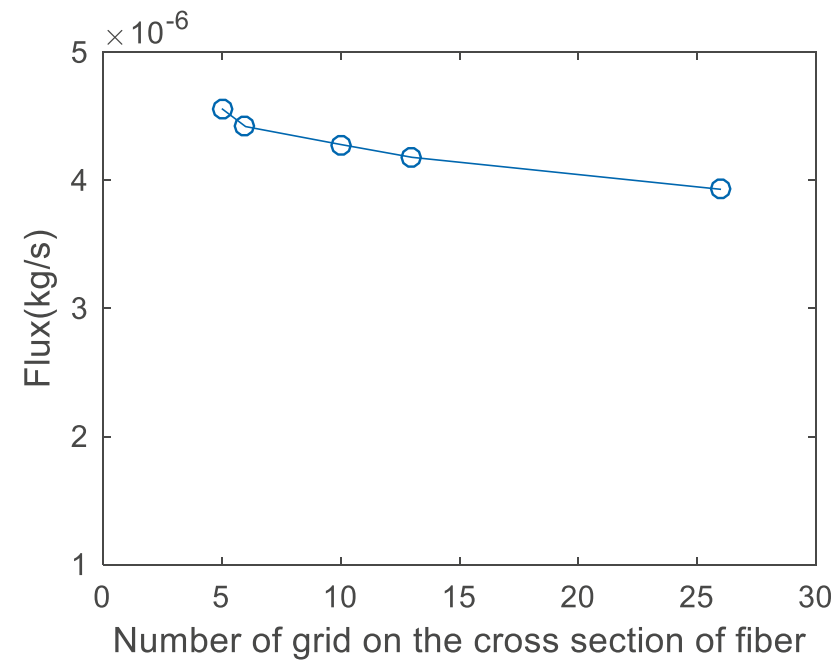

Fig. 6 Effect of mesh density on flux simulation

that there is an about $12 \%$ deviation between the flux values obtained when the grid size is increased from 5 to 25 . The deviation is reduced to about $5 \%$ when the mesh density is increased to 13 .

This result corroborates with a previous study [34] which found the influence of image resolutions on the permeability calculated using the Lattice Boltzmann method, similar to the influence of mesh density on the precision of FVM, was not strong. Moreover, the meshes are constructed as inscribed polygon in the fibers. Therefore, the porosity of virtual model composed of volume meshes will increase if the number of edges in the inscribed polygon decreases, and contributes to the increase of flux. Finally, to efficiently capture the velocity gradients in region near the fiber-fluid interface, about 20 mesh points are considered around the circular cross section of each fiber in this model. It is also reported by Tahir et al. [28] that there is no significant influence of permeability results for grid densities beyond 15 mesh points at the fiber cross section. Figure 5c, $\mathrm{d}$ shows the volume meshes. Consequently, the model is considered large enough to represent the macroscopic properties of fibrous media while being small enough to allow the computations to be carried out on a personal computer.

\subsection{Comparison with experimental data}

Permeability calculations for parallel flow (flow parallel to the material plane) taking $x$-axis as the representative direction and the transverse flow (flow perpendicular to the material plane) in $z$-axis direction are both investigated for PMFSSs with 80 and $90 \%$ porosities. The data of pressure gradient versus superficial velocity are shown in Fig. 7.

It can be seen that for the same pressure gradient, the fluid velocity increases with the increase of porosity, as 


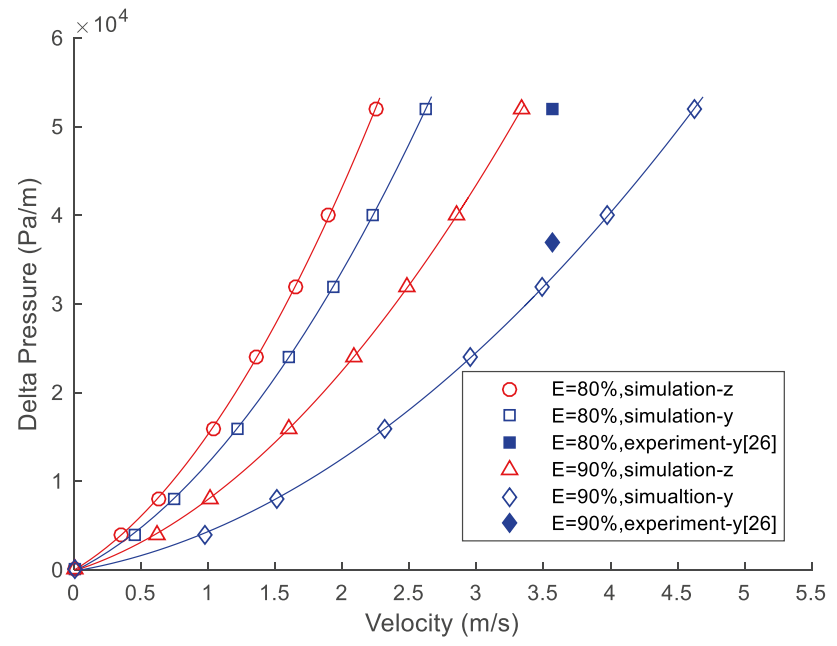

Fig. 7 Pressure gradient versus velocity curves of PMFSSs of 80 and $90 \%$ porosities with both parallel and transverse flows are investigated

Table 1 Results of permeability calculation

\begin{tabular}{lll}
\hline & \multicolumn{2}{l}{ Dimensionless permeability $\left(\mathrm{k} / \mathrm{r}^{2}\right)$} \\
\cline { 2 - 3 } & Parallel flow $(x$-direction) & $\begin{array}{l}\text { Transverse } \\
\text { flow }(z \text {-direc- } \\
\text { tion })\end{array}$ \\
\hline$E=90 \%$ & 2.570 & 1.372 \\
$E=80 \%$ & 0.884 & 0.732 \\
\hline
\end{tabular}

there is less drag force when flow passes through fewer fibers. In addition, for the same porosity, the calculated velocity of parallel flow is bigger than the one in the transverse flow direction, which reflects the influence of fiber alignment on permeability. The experimental data of parallel flow obtained previously published [33] show a better corroboration with the fitting curve for PMFSS with $90 \%$ porosity. However, the experimental data are about $40 \%$ higher than the calculated velocity of the same pressure for PMFSS with $80 \%$ porosity [(3.57-2.5)/2.5=40\%]. It is noteworthy that the accuracy of the experimental data is critically depended on the sensitivity of the equipment setting-up. Particularly, the experimentally measured flow flux according to certain pressure drop would be overestimated if there are enough gaps between the experimental flow container and porous media parallel in the flow direction. The simulated data are later fitted using the second-order polynomials. The fitting curves confirm that the Forchheimer law dominates the flow behavior at higher flow rate. At last, the permeability $k$ was obtained by fitting the data according to the Forchheimer equation (Eq. 5). The dimensionless permeability at different flow directions and porosities was summarized in Table 1.

\subsection{Comparison with the analytical models}

To determine the reliability of the simulations, the results are also compared with analytical models of Spielman and Goren [29]. Using Brinkman's equation, a group of permeability prediction models for fibrous networks with various fiber alignments is proposed. The equations they derived for flows in isotropically arranged structure Eq. (8), transverse Eq. (9), and parallel Eq. (10) flows for layered fiber arrangement are as follows:

$\frac{1}{4 \phi}=\frac{1}{3}+\frac{5}{6} \frac{\sqrt{k}}{r} \frac{K_{1}(r / \sqrt{k})}{K_{0}(r / \sqrt{k})}$

$\frac{1}{4 \phi}=\frac{1}{2}+\frac{\sqrt{k}}{r} \frac{K_{1}(r / \sqrt{k})}{K_{0}(r / \sqrt{k})}$

$\frac{1}{4 \phi}=\frac{1}{4}+\frac{3}{4} \frac{\sqrt{k}}{r} \frac{K_{1}(r / \sqrt{k})}{K_{0}(r / \sqrt{k})}$.

In these equations, $K_{0}$ and $K_{1}$ are the modified Bessel function of the second kind. Moreover, Davies [30] also gave the empirical correlation for layered structures:

$\frac{k}{r^{2}}=\left[16 \phi^{1.5}\left(1+56 \phi^{3}\right)\right]^{-1}$.

It is noteworthy that some other analytical models have also been proposed, such as predictions proposed by Tomadakis [31] and Drummond [32]. However, these models either under-predict or over-predict permeability in fibrous media, as discussed in [17], and thus, they were not compared in this work.

It can be seen in Fig. 8 that a good corroboration is obtained between the courses of the data of Davies's layered model (Eq. 11) and that of Spielman and Goren's layered model (Eq. 9).

A more detailed observation reveals that for the same porosity, the parallel permeability is bigger than the transverse permeability. This observation indicates that both fiber orientation and flow direction can affect the transport properties. The permeability results of PMFSSs with 80 and $90 \%$ porosities in parallel flow corroborate with the analytical equation for parallel flow (Eq. 10). The permeability of PMFSS of 90\% porosity in transverse flow is consistent with the analytical layered transverse model (Eqs. 9, 11). However, the simulated permeability of PMFSS with $80 \%$ porosity deviated from Eq. 9. The deviation can be attributed to fiber orientation. It should be noted that during model fitting, the fitting value of parameter $\beta$ of PMFSS with $80 \%$ porosity is 2.7 , which is less than the fitting value of PMFSS with $90 \%$ porosity (5.4). It indicates that the fiber arrangement for PFMSS with $80 \%$ porosity is more 


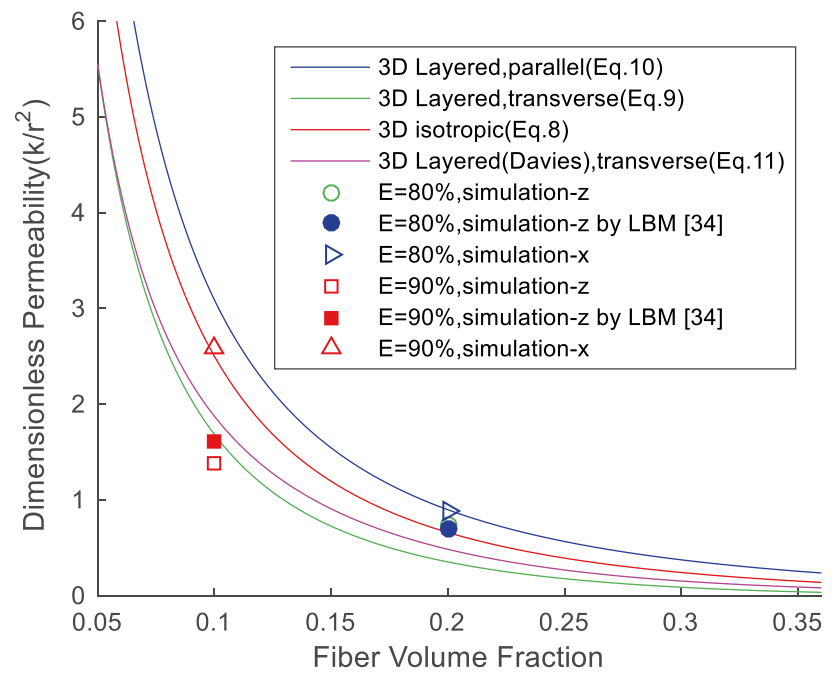

Fig. 8 Comparison of permeability obtained through simulation and analytical calculation

random and is closer to the isotropic orientation, consequently, with both the simulated parallel and transverse permeability of PMFSS with $80 \%$ porosity being approximate to the isotropic analytical model (Eq. 8). Another possible reason is the effect of bending of fibers in the virtual model, and therefore, a more random orientation is generated. At last, a good consistency is observed for the transverse permeability obtained using different simulation methods (FVM and LBM [34]). It indicates that both methods are applicable for flow study in complex structures and, meanwhile, verifies the reliability of our virtual modeling method.

\section{Conclusions}

In this paper, a 3D virtual modeling method and permeability calculations within PMFSSs are developed. The microstructure is studied by employing X-ray tomography and the morphology study is performed based on the skeleton representation. The 3D virtual model is constructed by taking account of the morphology characteristics of real materials, especially the fiber orientations estimated from the length-weighted fiber segment orientations. Using the virtual model, the transport property in the gaps between fibers is studied using finite volume method. Simulation results show that the simulated pressure gradient versus velocity data obeys the Forchheimer's law at a higher flow rate. The values of simulated permeability in both parallel and transverse directions are confirmed to be consistent with the analytical models proposed by Spielman and Goren [29]. It is concluded that both porosity and fiber arrangement have significant impacts on the transport properties, which provides useful suggestions in fiber arrangements and flow direction selections to achieve the optimization design and application of fibrous porous media. The proposed method is feasible for effective and efficient construction and simulation of fiber structure, hence providing support for optimal fiber structure design.

Acknowledgements This work is financially supported by the Nature Science Foundation of China (Grant Nos. 51505426 and 51275177), and the Open Fund of Key Ministry of Education \& Zhejiang Province Laboratory of E\&M (Zhejiang University of Technology, Grant No. 2014EP0012).

Open Access This article is distributed under the terms of the Creative Commons Attribution 4.0 International License (http:// creativecommons.org/licenses/by/4.0/), which permits unrestricted use, distribution, and reproduction in any medium, provided you give appropriate credit to the original author(s) and the source, provide a link to the Creative Commons license, and indicate if changes were made.

\section{References}

1. Tang Y, Zhou W, Xiang JH, Liu WY, Pan MQ (2010) An innovative fabrication process of porous metal fiber sintered felts with three-dimensional reticulated structure. Mater Manuf Process 25:565-871

2. Huang X, Wang QH, Zhou W, Li JR (2013) A simple fracture energy prediction method for fiber network based on its morphological features extracted by X-ray tomography. Mater Sci Eng A 585:297-303

3. Yuan W, Tang Yang, Yang XJ, Wan ZP (2012) Porous metal materials for polymer electrolyte membrane fuel cells-a review. Appl Energ 94:309-329

4. Yi PY, Pen LF, Lai XM, Li MT, Ni J (2012) Investigation of sintered stainless steel fiber felt as gas diffusion layer in proton exchange membrane fuel cells. Int J Hydrogen Energ 37:334-344

5. Magnus R, Erich S, Kararina L et al (2016) Computational highthroughput screening of fluid permeability in heterogeneous fiber materials. Soft Matter 12:6293-6299

6. Stylianopoulos T, Yeckel A, Derby JJ, Luo XJ, Shephard MS, Sander EA, Barocas VH (2008) Permeability calculations in three-dimensional isotropic and oriented fiber networks. Phys Fluids 20:123601

7. Nabovati A, Llewellin EW, Sousa ACM (2009) A general model for the permeability of fibrous media based on fluid flow simulations using the lattice Boltzmann method. Compos Part A 40:860-869

8. Vicente J, Daurelle JV, Brossard G, Blom A, Douteur A, Delmotte Y, Brun E (2012) Impact of different confluent fluid streams viscosities on interconnected porous static mixer device. Chem Eng Sci 72:172-178

9. Vicente J, Topin F, Daurelle JV (2006) Open celled material structural properties measurement: from morphology to transport properties. Mater Trans 47:2195-2202

10. Faessel M, Delisee C, Bos F, Castera P (2005) 3D modeling of random cellulosic fibrous networks based on X-ray tomography and image analysis. Compos Sci Technol 65:1931-1940

11. Pradeep B, Clinton SW, Karsten F (2011) Effect of network structure on characterization and flow modeling using $\mathrm{x}$-ray micro-tomography images of granular and fibrous porous media. Transp Porous Med 90:361-391 
12. Jeff T, Marios AI, Michael WF et al (2007) Pore network modeling of fibrous gas diffusion layers for polymer electrolyte membrane fuel cells. J Power Source 173:277-290

13. Qingrong X, Todor GB, Andrey PJ (2016) Review of pore network modelling of porous media: experimental characterisations, network constructions and applications to reactive transport. J Contam Hydrol 192:101-117

14. Sima D, Arash A, Yan W, Tequila H (2014) Modeling of composite fibrous diffusion media. Int $\mathrm{J}$ Hydrogen Energ 39:9375-9386

15. Koivu V, Decain M, Geindreau C, Mattila K, Bloch JF, Kataja M (2009) Transport properites of heterogeneous materials. Combing computerised X-ray micro-tomography and direct numerical simulations. Int J Comput Fluid D 23:713-721

16. Brun E, Vicente J, Topin F, Occelli R, Clifton MJ (2009) Microstructure and transport properties of cellular materials: representative volume element. Adv Eng Mater 11:805-810

17. Jaganathan S, Tafreshi HV, Pourdeyhimi B (2008) A realistic approach for modeling permeability of fibrous media: 3-D imaging coupled with CFD simulation. Chem Eng Sci 63:244-252

18. Heitzmann R, Tempfli E, Schmidt FP (2015) Investigation of the permeability of anisotropic fibre structures through CFD simulation. Transp Porous Med 108:313-333

19. Shou D, Fan J, Ding F (2011) Hydraulic permeability of fibrous porous media. Int J Heat Mass Transf 54:4009-4018

20. Perona P, Malik J (1990) Scale-space and edge detection using anisotropic diffusion. IEEE Trans pattern Anal Mach Intell 12:629-639

21. Lorensen WE, Cline HE (1978) Marching cubes: a high resolution 3D surface construction algorithm. Comput Graph 21:163-169

22. Tan JC, Elliott JA, Clyne TW (2006) Analysis of tomography images of bonded fibre networks to measure distributions of fibre segment length and fibre orientation. Adv Eng Mater 8:495-500

23. Tan JC, Clyne TW (2008) Ferrous fibre network materials for jet noise reduction in aeroengines Part 2: Thermo-mechanical stability. Adv Eng Mater 10:201-209
24. Redenbach C, Vecchio I (2011) Statistical analysis and stochastic modeling of fibre composites. Compos Sci Technol 71:107-112

25. Bonnet JP, Topin F, Tadrist L (2008) Flow laws in metal foams: compressibility and pore size effects. Transp Porous Med 73:233-254

26. Clague DS, Kandhai BD, Zhang R, Sloot PMA (2000) Hydraulic permeability of (un) bounded fibrous media using the lattice Boltzmann method. Phys Rev E 61:616-625

27. Jackson GW, James DF (1986) The permeability of fibrous porous media. Can J Chem Eng 64:364-374

28. Tahir MA, Tafreshi HV (2009) Influence of fiber orientation on the transverse permeability of fibrous media. Phys Fluids 21:083604

29. Spielman L, Goren SL (1986) Model for predicting pressure drop and filtration efficiency in fibrous media. Environ Sci Technol 2:279-287

30. Davies CN (1953) The separation of airborne dust and particles. Proc Inst Mech Eng B J Eng Manuf 1(1-12):185-213

31. Tomadakis MM, Robertson JT (2005) Viscous permeability of random fiber structures: comparison of electrical and diffusional estimates with experimental and analytical results. J Compos Mater 39(2):163-188

32. Drummond JE, Tahir MI (1984) Laminar viscous flow through regular arrays of parallel solid cylinders. Int $\mathrm{J}$ Multiph Flow 10(3):515-540

33. Zhou W, Wang QH, Qiu QF, Tang Y et al (2015) Heat and mass transfer characterization of porous copper fiber sintered felt as catalyst support for methanol steam reforming. Fuel $145: 136-142$

34. Huang X, Wang QH, Zhou W, Deng DX et al (2015) Morphology and transport properties of fibrous porous media. Powder Technol 283:618-626

35. Qinghui W, Xiang H, Wei Z et al (2013) Three dimensional reconstruction and morphologic characteristics of porous metal fiber sintered sheet. Mater Charact 86:49-58 